\title{
ROLE OF TEST SEQUENCE LENGTH IN SOFTWARE TESTING FOR STRUCTURAL COVERAGE-AN EMPIRICAL PROOF
}

\author{
M. Vijaya Bharathi ${ }^{1}$ and Sireesha Rodda ${ }^{2}$ \\ ${ }^{1}$ CSE Department, BIET Mangalpally \& Research Scholar @ GITAMU \\ ${ }^{2}$ Professor CSE Department, GITAM University, Vishakhapatnam, Andhra \\ Pradesh, India \\ 1mvbharathi328@gmail.com
}

\begin{abstract}
Testing is a basic period of programming advancement life cycle. This work goes for investigating the part that the length of test successions plays in testing programming. Specifically, we focus on the branch scope paradigm. Code scope is a measure used to depict how much the source code of a program is executed when a specific test suite runs. To acquire high scope a typical practice is to apply a first period of arbitrary testing, trailed by more modern methods went for covering the rest of the branches. we dissect just this second stage. For each troublesome branch we examine the errand of finding a solitary and insignificant succession to cover it. Indeed, to cover a specific branch of the code, an arrangement of past capacity calls may be required to put the inward state in the suitable design. Here we have taken one illustration program and composed diverse length test arrangements for that. We tended to the distinction in scope when diverse length test arrangements are utilized. To test the program, we have utilized mechanized devices JUnit for segment testing. When it is over, utilizing EclEmma the scope of experiments is assessed and demonstrated enhanced arrangements are ideal.
\end{abstract}

Keywords - test sequence, coverage, empirical

\section{INTRODUCTION}

Testing is a fundamental period of programming advancement life cycle and it is basic to reveal the bugs in the product. There are many trying strategies yet the thorough testing is outlandish for each product. We can state testing a product is only checking the every single line of code in the event that we can get most extreme scope then that testing procedure will uncovers more number of bugs.

Test Case: An experiment is an arrangement of conditions or factors under which an analyzer will decide if a framework under test fulfills prerequisites or works accurately. The way toward creating test cases can likewise help discover issues in the necessities or outline of an application.

TEST SUITE: The route in which test cases are assembled in light of module savvy and sub module insightful structure alongside components.

TEST PLAN: It is a report comprising of finish testing movement for a fruitful conveyance of a extend.

TEST SCRIPT: When the experiments are robotized by utilizing computerization instrument it is called as Test Script.

Received: November 26, 2018

Reviewed: January 28, 2019

Accepted: February 4, 2019 
TEST SEQUENCE: Sequence of request of related activity or steps that contain a test methodology or trial. An experiment is a driver that calls the capacity under test with a specific arrangement of information qualities. The driver then looks at the acquired yield against the normal one. Utilizing every single conceivable info is infeasible in light of the fact that their number is unbounded as a rule. Subsequently, the robotization of programming testing in this setting comprises of naturally finding the littlest arrangement of these sources of info with the end goal that the testing model is boosted. On another obscure testing issue, we would not know what number of capacity calls we have to acquire full scope. In a perfect world, the briefest length ought to be favored. In this work, we break down the part that the length of test arrangements plays in testing programming with interior state. Specifically, we focus on the branch scope foundation since it is a standout amongst the most well-known criteria in the writing. To acquire high scope, a typical practice is to apply a first period of arbitrary testing, trailed by more modern methods went for covering the rest of the branches. For each troublesome branch we break down the errand of finding a solitary and negligible grouping to cover it. Since infeasible branches will never be secured.

Different Types of Testing: Unit Testing: It is a kind of testing of an individual unit or gathering of related units. It falls under the class of white box testing. It is regularly done by the software engineer to test that the unit they has executed is creating expected yield against given info.

Joining Testing: It is a kind of testing in which a gathering of segments are joined to deliver yield. Additionally, the cooperation amongst programming and equipment is tried in coordination testing. It might fall under both white box testing and discovery testing.

Practical Testing: It is a sort of testing which confirms that each capacity of the product application works in conformance with the prerequisite particular. Every single usefulness of the framework is tried by giving suitable info. It might fall under discovery testing.

Framework Testing: It is the trying to guarantee that by putting the product in various situations (e.g., Operating Systems) regardless it works. It falls under the class of discovery testing.

Push Testing: It is a sort of testing to assess how framework carries on under troublesome conditions. Testing is directed at past points of confinement of the determinations. It falls under the class of discovery testing.

Execution Testing: It is a sort of testing to survey the speed and adequacy of the framework and to ensure it is producing comes about inside a predetermined time as in execution prerequisites. It falls under the class of discovery testing.

Convenience Testing: It is performed to the viewpoint of the customer, to assess how the GUI is easy to understand? How effectively can the customer learn? In the wake of figuring out how to utilize, how capably can the customer perform? This falls under the class of discovery testing.

Acknowledgment Testing: Acknowledgment testing is frequently done by the client to guarantee that the conveyed item meets the necessities and fills in as the client anticipated. It falls under the class of discovery testing.

Relapse Testing: Relapse testing is the trying after alteration of a framework, part, or a gathering of related units to guarantee that the adjustment is working accurately and is not harming or forcing different modules to deliver sudden outcomes. It falls under the class of discovery testing.

Beta Testing: Beta testing is the trying which is finished by end clients, a group outside improvement, or openly discharging full pre-adaptation of the item which is known as beta variant. The point of beta testing is to cover startling blunders. It falls under the class of discovery testing. 


\section{Measures of Software Testing Completeness:}

- $\quad$ Requirements Coverage:

Has the software been tested against all requirements for the normal range of use?

Has the software been tested against all requirements for abnormal or unexpected usage?

- $\quad$ Structural Coverage: Has each element of the software been exercised during testing?

- $\quad$ Architectural Coverage: Have the actual control and data links been utilized during testing?

\section{Structural (code) Coverage:}

It is to measure what percentage of code has been exercised by a test sequence. Coverage criteria are usually defined as a rule or requirement, which test suite needs to satisfy.

\section{Basic coverage criteria:}

There are a number of coverage criteria, the main ones being

Function coverage - $\quad$ Has each function in the program been called?

Statement coverage - Has each statement in the program been executed?

Branch coverage - $\quad$ Has each branch of each control structure been executed? For example, given an if statement, have both the true and false branches been executed? Another way of saying this is, has every edge in the program been executed?

Condition coverage - $\quad$ Has each Boolean sub-expression evaluated both to true and false?

Assume this function is a part of some bigger program and this program was run with some test suite.

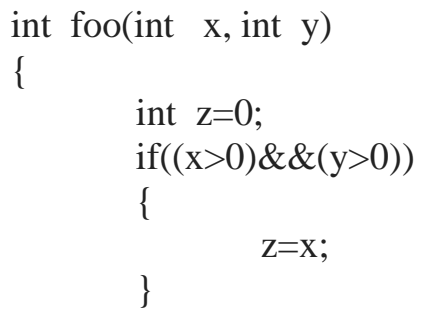

- If during this execution function 'foo' was called at least once, then Function coverage for this function is satisfied.

- $\quad$ Statement coverage for this will be satisfied if it have test sequence as foo $(1,1)$, as in this case, every line in the function is executed including $\mathrm{z}=\mathrm{x}$;

- Tests calling foo $(1,1)$ and foo $(0,1)$ will satisfy Branch coverage because, in the first case, the 2 if conditions are met and $\mathrm{z}=\mathrm{x}$; is executed, while in the second case, the first condition $(x>0)$ is not satisfied, which prevents executing $z=x$;

- Condition coverage can be satisfied with tests that call foo $(1,0)$ and foo $(0,1)$. These are necessary because in the first cases, $(x>0)$ evaluates to true, while in the second, it evaluates false. At the same time, the first case makes $(y>0)$ false, while the second makes it true.

\section{LITERATURE SURVEY}

In 2012 in research article "A Theoretical and Empirical Analysis of the Role of Test Sequence Length in Software Testing for Structural Coverage" by Dr. Andrea Arcuriin IEEE Computer Society, May/June 2009 Volume 38 Number 3explained about that test 
grouping length assumes a vital part in programming testing with the end goal that more drawn out test arrangements can give preferred scope over shorter.

Within the sight of an inside state, regularly a grouping of capacity calls is required to test programming. Truth be told, to cover a specific branch of the code, an arrangement of past capacity calls may be required to put the interior state in the suitable setup. In this paper, he dissect the part that the length plays in programming testing, specifically branch scope. He demonstrates that, on "troublesome" programming testing benchmarks, longer test successions make their testing inconsequential. Henceforth, we contend that the decision of the length of the test arrangements is critical in programming testing.

In this paper, examined the part that the length of test successions plays in programming testing for basic scope. We have exactly appeared on regular programming testing benchmarks that having marginally longer arrangements can definitely enhance the outcomes; likewise specified future work will take after two headings. Initially, practical programming for which longer groupings give more awful outcomes should be recognized and considered.

In 2015 research article "Random Testing: The Best Coverage Technique - An Empirical Proof" by K Koteswara Rao and Prof GSVP Raju propose a hypothetically and inferred that the execution of irregular testing is exceptionally great when it is contrasted and parcel testing despite the fact that it gives great outcomes for testing. Here in this paper we tended to the distinction in scope between irregular testing and parcel testing, here we have taken one case program and composed the experiments for that.

Irregular testing is especially helpful system in creating arbitrary contributions with these arbitrary information sources the experiments will cover more number of lines in the source code. The experiments with more scope procedures better test comes about due the ignorance on arbitrary testing it is not utilized as a part of numerous applications testing.

In 2013 research article "Effect of Length of Test Sequence on Software Coverage" by B. Raveendranath Singh suggested that the length of test successions so as to guarantee finish basic scope in the product is the range less considered. They goes for breaking down the part of length of test groupings for finish branch scope.

By utilizing the pursuit procedures, for example, RS, HC, EA, GA and different varieties of GA. He have tried compartments and other programming items utilizing a model application. The application is fabricated utilizing Java dialect keeping in mind the end goal to test programming in easy to use mold. The product under test contains interior state also. With inward state, he made analyses with different strategies keeping in mind the end goal to locate the ideal test succession length. He found that test succession length assumes a significant part in programming testing for finish basic scope. The trial comes about uncovered that the proposed application is helpful in testing programming and produce test cases for full branch scope.

In 2010, IEEE International Conference "Are Longer Test Sequences Always Better? A Reliability Theoretical Analysis" by Fevzi Belli, Nevin Güler, Michael Linschulte emerges the one of the fascinating inquiries as of now talked about in programming testing, both by and by and the scholarly community, is the part of test groupings on programming testing, particularly on blame discovery. Conviction is "the more extended the better", i.e., the more extended test groupings are, the more blames are identified.

At long last they have dissected the blame discovery probability of test successions of expanding length which are created and chosen by methods for extensive measured occasion grouping diagrams (ESG). In a test situation, various tests are then methodically and consequently did on a huge online traveler reservation framework called ISELTA. The test information acquired have been gathered for test lengths of 2, 3, 4, and 5, and after that dissected by methods for a progression of programming unwavering quality development models of various classes to maintain a strategic distance from potential misconceptions brought on by utilizing a solitary model. At last, assessed blame figures 
are contrasted with genuine figures conveyed by tests to discover how far and how precisely the model estimations correspond to the truth.

\section{STATEMENT OF THE PROBLEM}

Testing is a basic period of programming advancement life cycle. This work goes for breaking down the part that the length of test groupings plays in testing programming. Specifically, we focus on the branch scope model. Code scope is a measure used to depict how much the source code of a program is executed when a specific test suite runs. A program with high code scope, measured as a rate, has had a greater amount of its source code executed amid testing which recommends it has a lower shot of containing undetected programming bugs contrasted with a program with low code scope. Here we have taken one case program and composed distinctive length test arrangements for that. We tended to the distinction in scope when diverse length test arrangements are utilized. To test the program we have utilized mechanized devices JUnit for parcel testing. When it is over, utilizing EclEmma the scope of experiments is assessed and demonstrated improved successions are ideal.

\section{TECHNICAL DESCRIPTION:}

Overshadow: It is a coordinated advancement condition (IDE) utilized as a part of PC programming and is the most generally utilized Java IDE. It contains a base workspace and an extensible module framework for tweaking the earth. Shroud is composed for the most part in java and its essential utilize is for creating Java applications, however it might likewise be utilized to create applications in other programming dialects through modules

JUnit: It is a unit testing system for Java programming dialect. JUnit has been critical in the improvement of test-driven advancement, and is one of a group of unit testing structures by and large known as xUnit, that began with JUnit.

EclEmma: It is an Eclipse module that creates code scope reports. We can undoubtedly ascertain scope for littler projects, be that as it may, for bigger projects, it is a great deal more troublesome so in such case EclEmma, help via robotizing the scope procedure and giving intelligible reports. EclEmma can be utilized with JUnit. It keeps running with the JUnit test cases and produces the scope report from the execution. It gives by and large, bundle, and record level data on articulation. It can be downloaded for a manual introduce from its Source Forge Project Page. Furthermore, we can likewise introduce EclEmma with obscuration. For more data on introducing shroud modules, counsel the instructional exercise regarding the matter.

Establishment of module:

1. From your Eclipse menu select Help $\rightarrow$ Install New Software...

2. In the Install exchange enter http://update.eclemma.org/at the Work with field.

3. Check the most recent EclEmma form and press Next

4. Take after the means in the establishment wizard. 


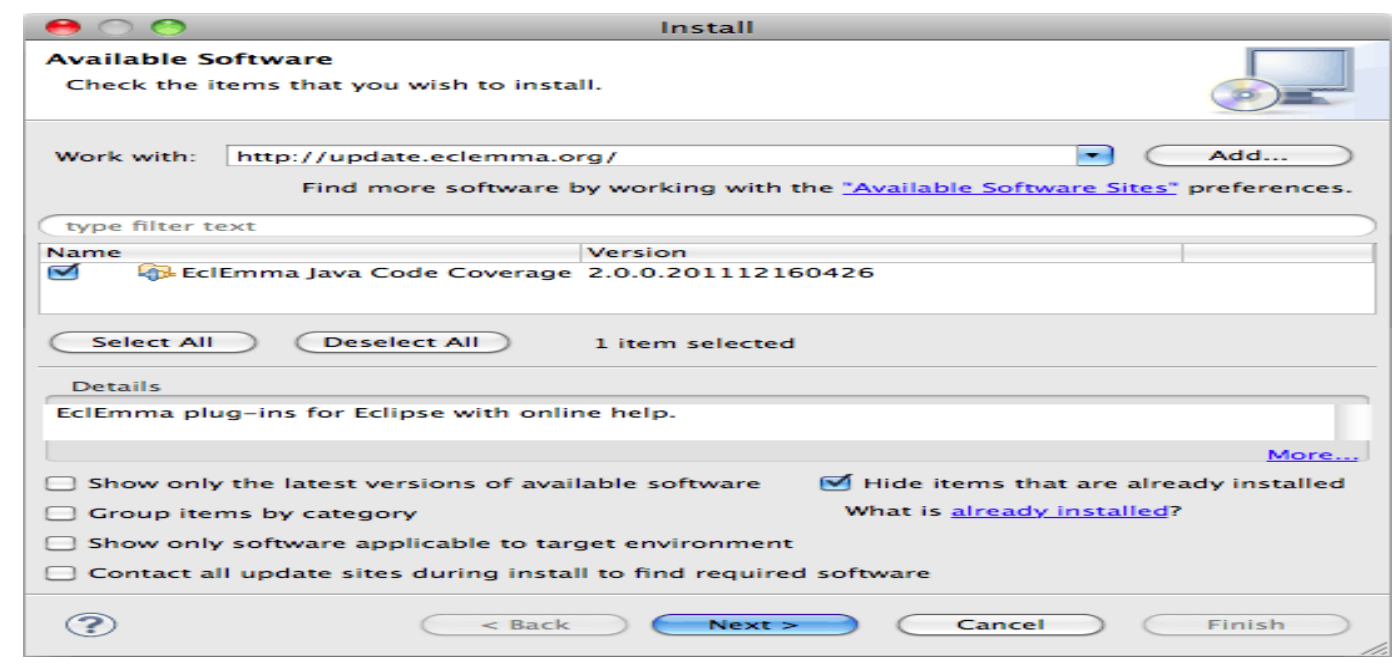

Fig. 4.b. 1:Installation of EclEmma plug-in

\section{PROBLEM METHODOLOGY AND SOLUTION}

In this we have taken a java program and composed distinctive length test arrangements for the program then computed the scope for each situation. In this we have chiefly 3 modules

1. Installation of module.

2. Writing Java program and Test Sequences.

3. Calculating Coverage.

1. Installation of plug-in: $\quad$ For detailed information about this refer chapter (4.b).

2. Writing Java program and Test Sequences:

\section{Simple java program:}

package project;

public class Interest \{

public static int calInterest(int year) \{

int $\mathrm{p}=1000$;

int $\mathrm{r}=0, \mathrm{a}=0$;

if $($ year $>=8)$

$\mathrm{r}=15$;

else if $($ year $>=6$ )

$$
r=10 \text {; }
$$

else if $($ year $>=3$ )

$$
r=5 \text {; }
$$

else if $($ year $>=1)$

$$
\mathrm{r}=3 \text {; }
$$

else

$r=0$;

if $(r>=3)$

$\mathrm{a}=(((\mathrm{p} * \mathrm{r} *$ year $) / 100)+\mathrm{p}) ;$

else

$$
\mathrm{a}=\mathrm{p}
$$

return $a$;

\} 
For this straightforward program we are composing distinctive length test arrangements. Here we are separating the test contribution to four subsets

1. Test with the run of the mill an incentive in the parcel

2. Test at the lower and higher limit values

3. Test at correct limits

4. Test without of bound esteem

Test sequence1 developed for Interest.java:

package project;

import junit.framework.TestCase;

public class InterestTest 3 extends TestCase \{

protected void setUp() throws Exception \{

super.setUp();

\}

protected void tearDown() throws Exception \{

\} super.tearDown();

public void testTypical() \{

assertEquals("wrong", 1030, Interest.calInterest(9));

assertEquals("wrong", 1200, Interest.calInterest(5));

assertEquals("wrong", 1880, Interest.calInterest(10));

assertEquals("wrong", 2659, Interest.calInterest(9));

assertEquals("wrong", 1030, Interest.calInterest(4));

assertEquals("wrong", 4000, Interest.calInterest(12));

assertEquals("wrong", 2900, Interest.calInterest(5));

\}

public void testNearBoundaries() \{

assertEquals("wrong", 1080, Interest.calInterest(5));

assertEquals("wrong", 1790, Interest.calInterest(4));

assertEquals("wrong", 1000, Interest.calInterest(6));

assertEquals("wrong", 1790, Interest.calInterest(5));

assertEquals("wrong", 8900, Interest.calInterest(9));

assertEquals("wrong", 1200, Interest.calInterest(5));

assertEquals("wrong", 0000, Interest.calInterest(6));

assertEquals("wrong", 8900, Interest.calInterest(9));

assertEquals("wrong", 8000, Interest.calInterest(9));

\}

public void testAtBoundaries() \{

assertEquals("wrong", 1031, Interest.calInterest(10)); assertEquals("wrong", 1601, Interest.calInterest(6));

assertEquals("wrong", 2501, Interest.calInterest(10));

assertEquals("wrong", 1151, Interest.calInterest(6));

assertEquals("wrong", 6400, Interest.calInterest(9));

assertEquals("wrong", 3400, Interest.calInterest(9));

assertEquals("wrong", 0000, Interest.calInterest(6));

assertEquals("wrong", 6000, Interest.calInterest(10));

assertEquals("wrong", 6000, Interest.calInterest(6));

\}

public void testAtOutOfBounds( $)$ \{

assertEquals("wrong", 1090, Interest.calInterest(23));

assertEquals("wrong", 4000, Interest.calInterest(30));

assertEquals("wrong", 3000, Interest.calInterest(45));

assertEquals("wrong", 0000, Interest.calInterest(16)); 


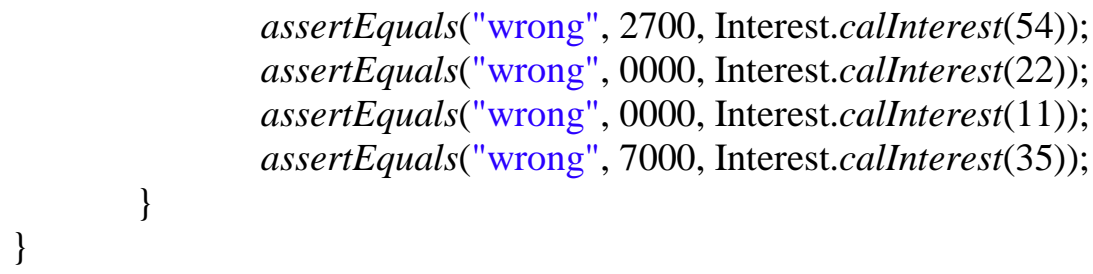

Test sequence 2 developed for Interest.java:

package project;

import junit.framework. TestCase;

public class InterestTest 2 extends TestCase \{

protected void $\operatorname{set} U p()$ throws Exception \{

super.setUp();

)

protected void tearDown() throws Exception \{

super.tearDown();

西

public void testTypical() \{ // test a typical(in between values) value in partitions

assertEquals("wrong", 1030, Interest.calInterest(2));

assertEquals("wrong", 1250, Interest.calInterest(5));

assertEquals("wrong", 1800, Interest.calInterest(8));

assertEquals("wrong", 2659, Interest.calInterest(9));

assertEquals("wrong", 1030, Interest.calInterest(1));

\}

public void testNearBoundaries() \{

assertEquals("wrong", 1030, Interest.calInterest(1)); assertEquals("wrong", 1080, Interest.calInterest(2)); assertEquals("wrong", 1700, Interest.calInterest(7)); assertEquals("wrong", 1000, Interest.calInterest $(0))$; assertEquals("wrong", 1790, Interest.calInterest(9));

) assertEquals("wrong", 0000, Interest.calInterest(11));

public void testAtBoundaries() \{

assertEquals("wrong", 1030, Interest.calInterest(1));

assertEquals("wrong", 1601, Interest.calInterest(6));

assertEquals("wrong", 1000, Interest.calInterest(0));

assertEquals("wrong", 2501, Interest.calInterest(10));

assertEquals("wrong", 1151, Interest.calInterest(3));

\}

public void testAtOutOfBounds() \{

assertEquals("wrong", 1090, Interest.calInterest(-1));

assertEquals("wrong", 4000, Interest.calInterest(20));

assertEquals("wrong", 3000, Interest.calInterest(15));

assertEquals("wrong", 0000, Interest.calInterest(-4));

assertEquals("wrong", 1000, Interest.calInterest(25));

assertEquals("wrong", 5500, Interest.calInterest(30));

assertEquals("wrong", 0000, Interest.calInterest(-2));

assertEquals("wrong", 1000, Interest.calInterest(34));

\}

Test sequence3 developed for Interest.java: 
package project;

import junit.framework. TestCase;

public class InterestTest 1 extends TestCase \{

protected void setUp() throws Exception \{

\} super.setUp();

protected void tearDown() throws Exception \{ super.tearDown();

\}

public void testTypical() \{ // test a typical(in between values) value in partitions assertEquals("wrong", 1250, Interest.calInterest(5)); assertEquals("wrong", 1800, Interest.calInterest(8)); assertEquals("wrong", 2650, Interest.calInterest(11));

\}

public void testNearBoundaries() \{

assertEquals("wrong", 1000, Interest.calInterest (0));

assertEquals("wrong", 1060, Interest.calInterest(2));

assertEquals("wrong", 1200, Interest.calInterest(4));

assertEquals("wrong", 1700, Interest.calInterest(7));

\}

public void testAtBoundaries() \{

assertEquals("wrong", 1030, Interest.calInterest(1));

assertEquals("wrong", 1150, Interest.calInterest(3)); assertEquals("wrong", 1100, Interest.calInterest(6)); assertEquals("wrong", 2500, Interest.calInterest(10));

\}

public void testAtOutOfBounds() \{

assertEquals("wrong", 1000, Interest.calInterest(-1));

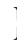

assertEquals("wrong", 4000, Interest.calInterest(20));

\}

The detailed procedure for creating test sequences using automated JUnit tool can be viewed in following steps .

Step1: Create a JUnit test case for Interest.java; you can see the following figure for creating test case for Interset.java

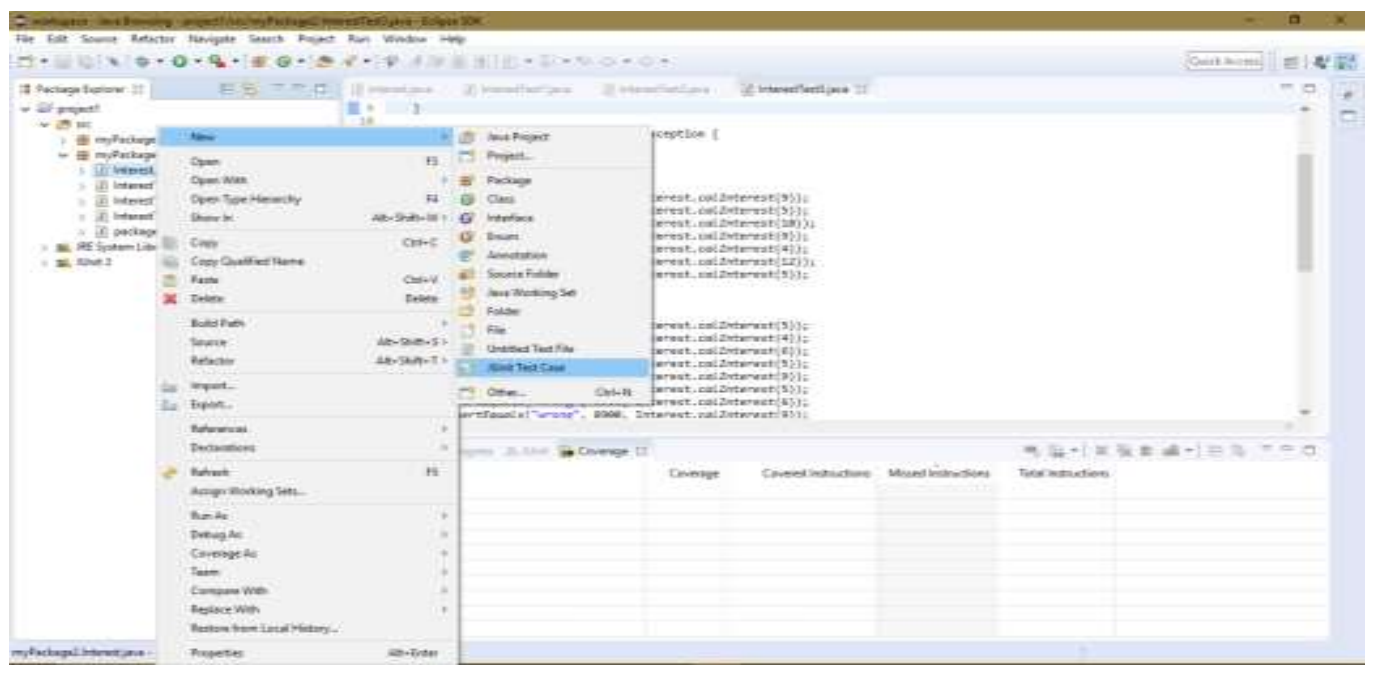

Fig. 5.b.1: Creating JUnit Test Cases 
Step2: Whenever we click on JUnit test case a new dialog box will be appeared to select the testing frame work you would like to test (i.e., JUnit 3 or JUnit 4) here we used JUnit 3.

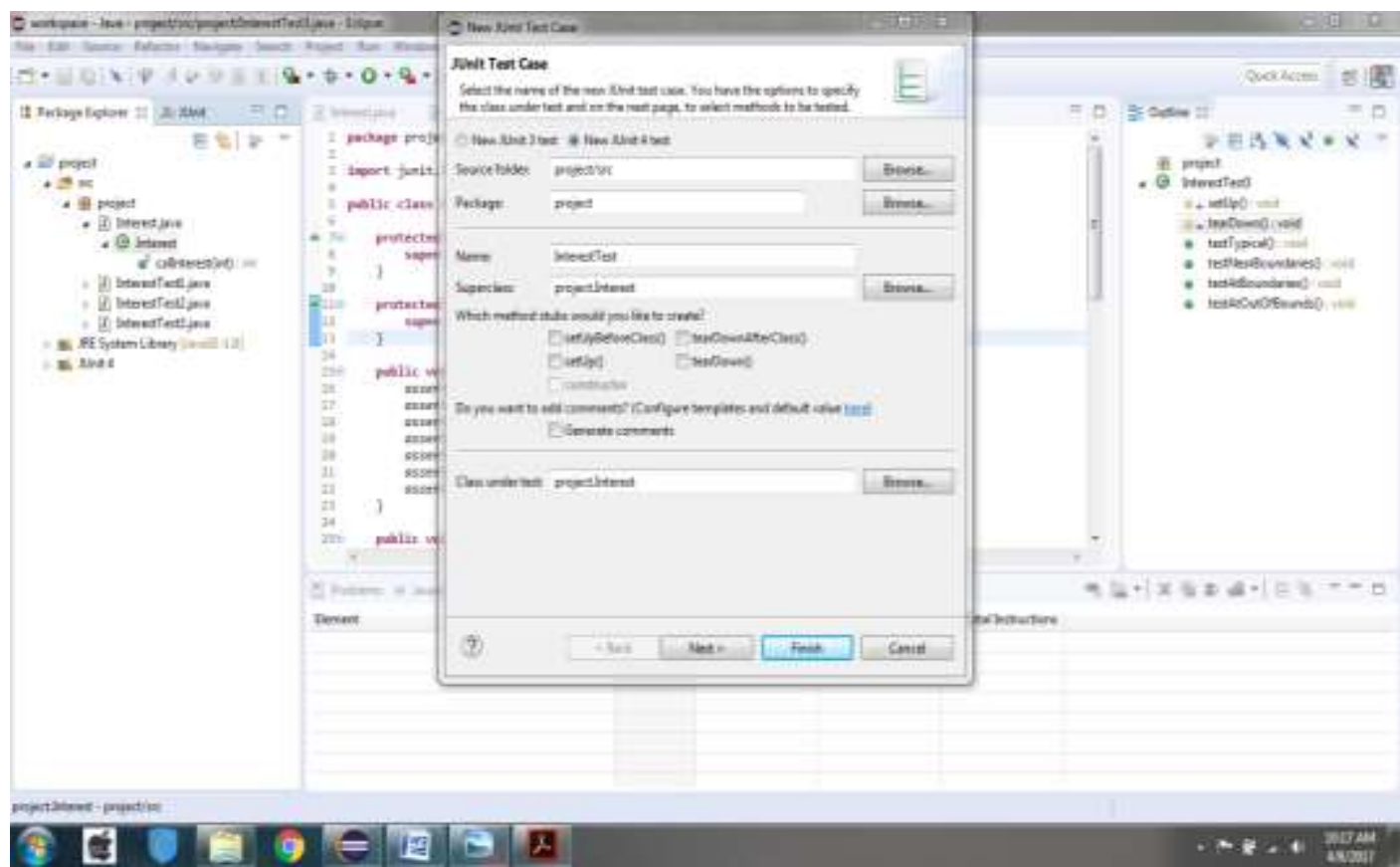

Fig. 5.b.2. Selecting Framework

Step 3: After selecting testing frame work it will be displayed as you can see in the following figure the JUnit 3 framework displayed for new test case

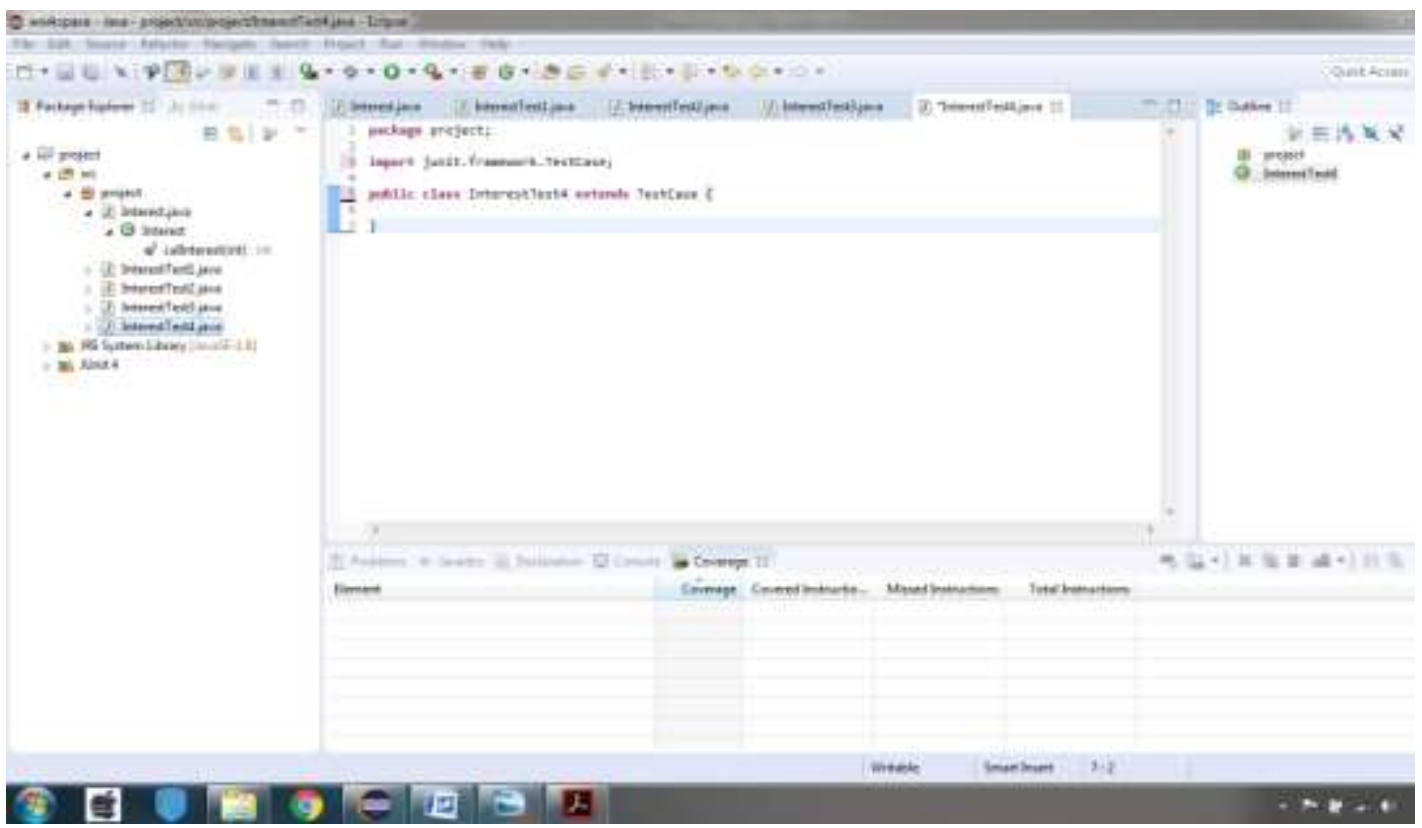

Fig. 5.b.3. Showing Selected Framework

Step 4: Write the manual test cases for all the input partitions that we are divided for the input domain (here for Interest .java we divided the input domain into 4 subsets). 


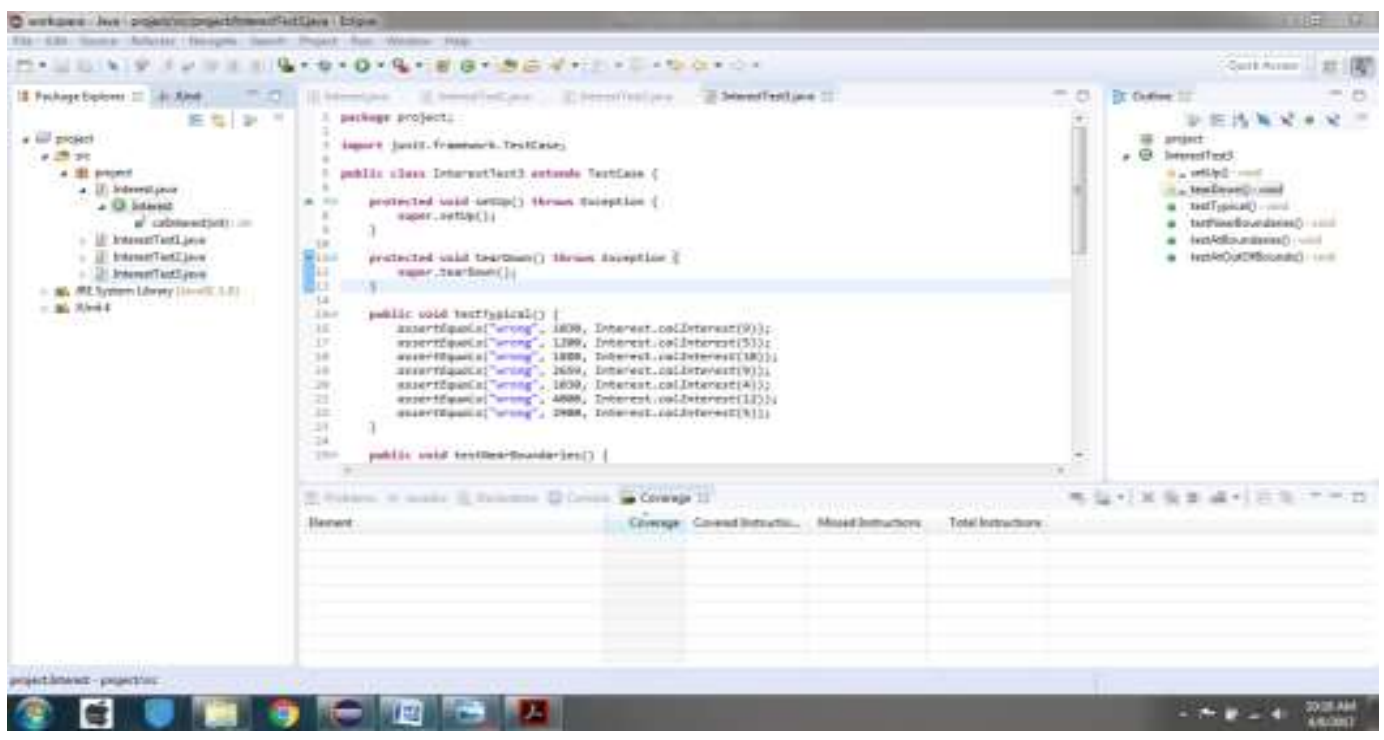

Fig. 5.b.4. Displaying Test Cases for the Interest Class

\section{CAlCulating COVERAge}

Once if we write test cases for all partitions run the test class and you can see passed and failure test cases after that click on EclEmma code coverage icon it will display the coverage as you can see in the following figure(coverage, Covered instructions, Missed Instructions, total instructions).

The coverage for Interest.java for first test sequence which has highest length is $69.8 \%$.

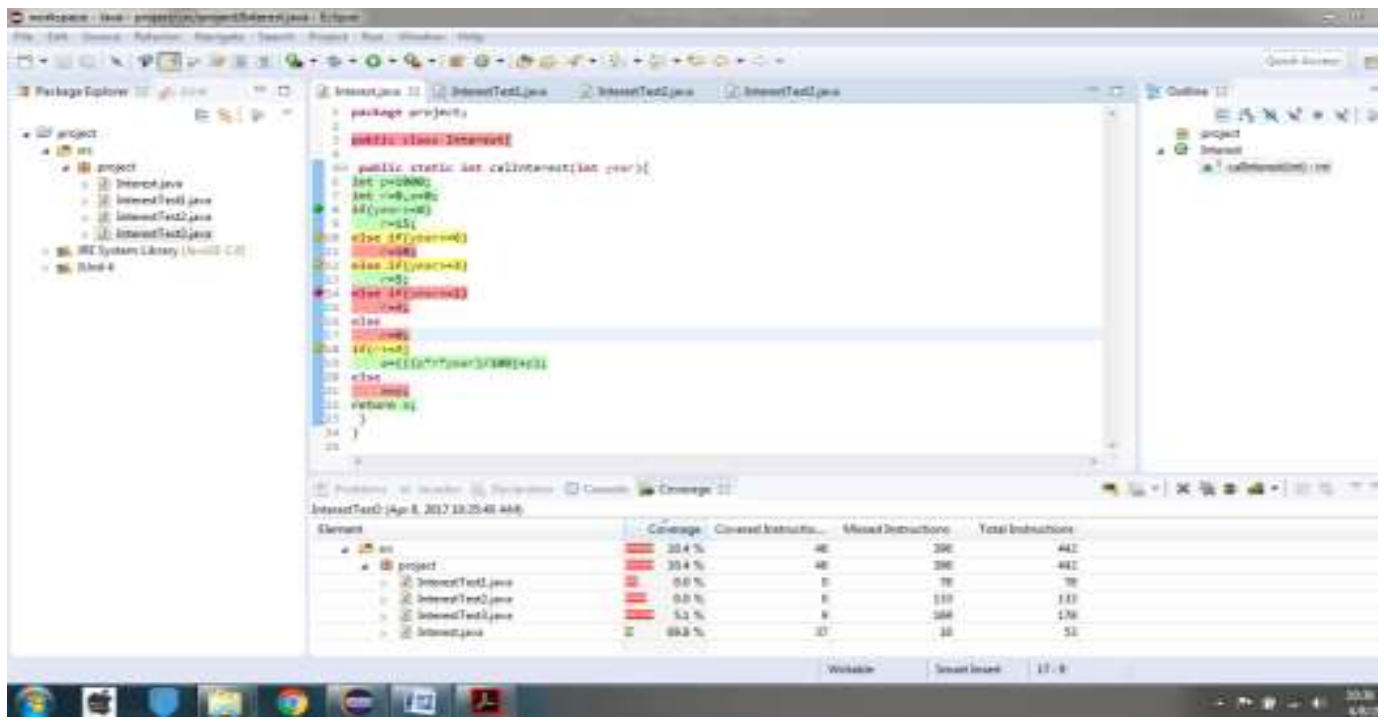

Fig. 5.c.1. Coverage for the Test Sequence InterestTest3.java

The coverage for Interest.java for first test sequence which medium length is $83.0 \%$. 


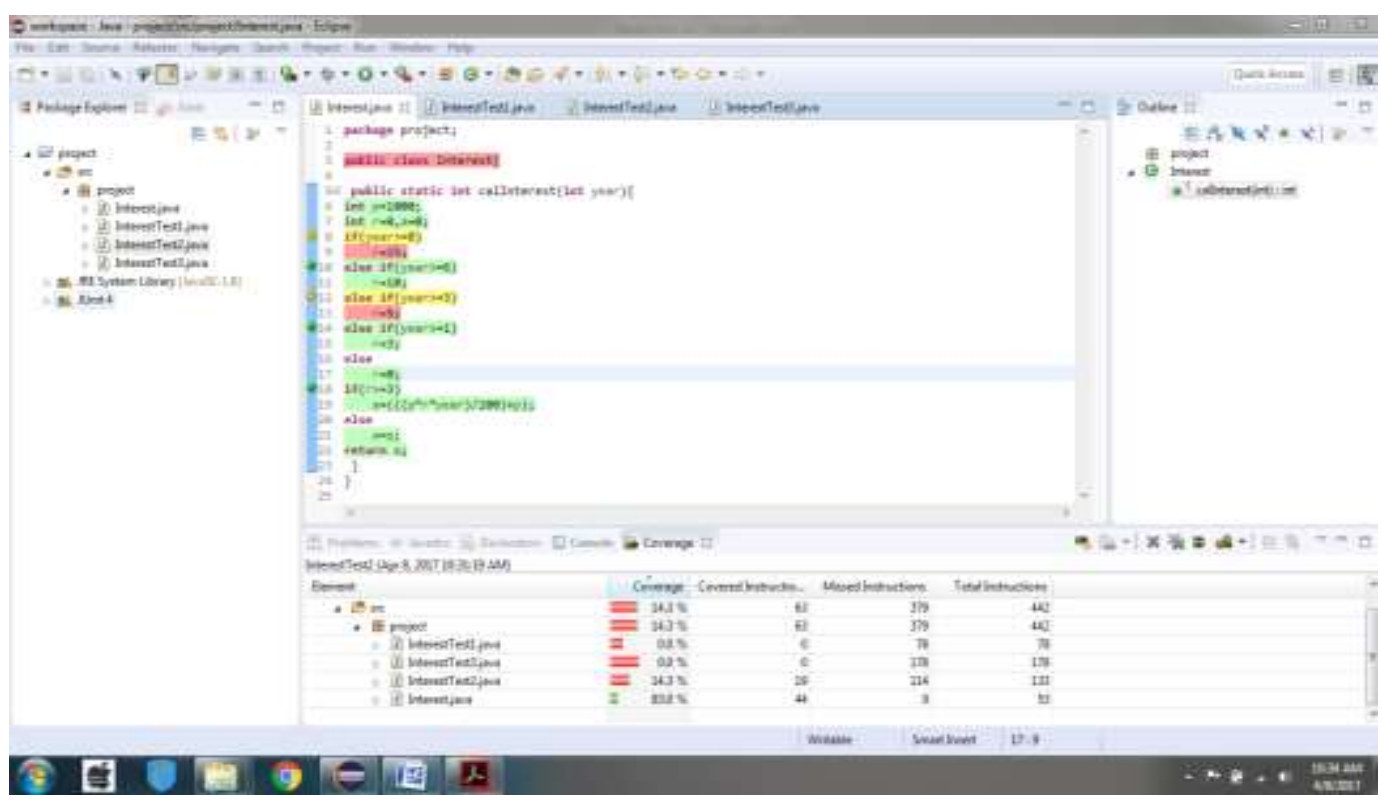

Fig. 5.c.2. Coverage for the Test Sequence InterestTest2.java

The coverage for Interest.java for first test sequence which has minimum length is 93.4\%.

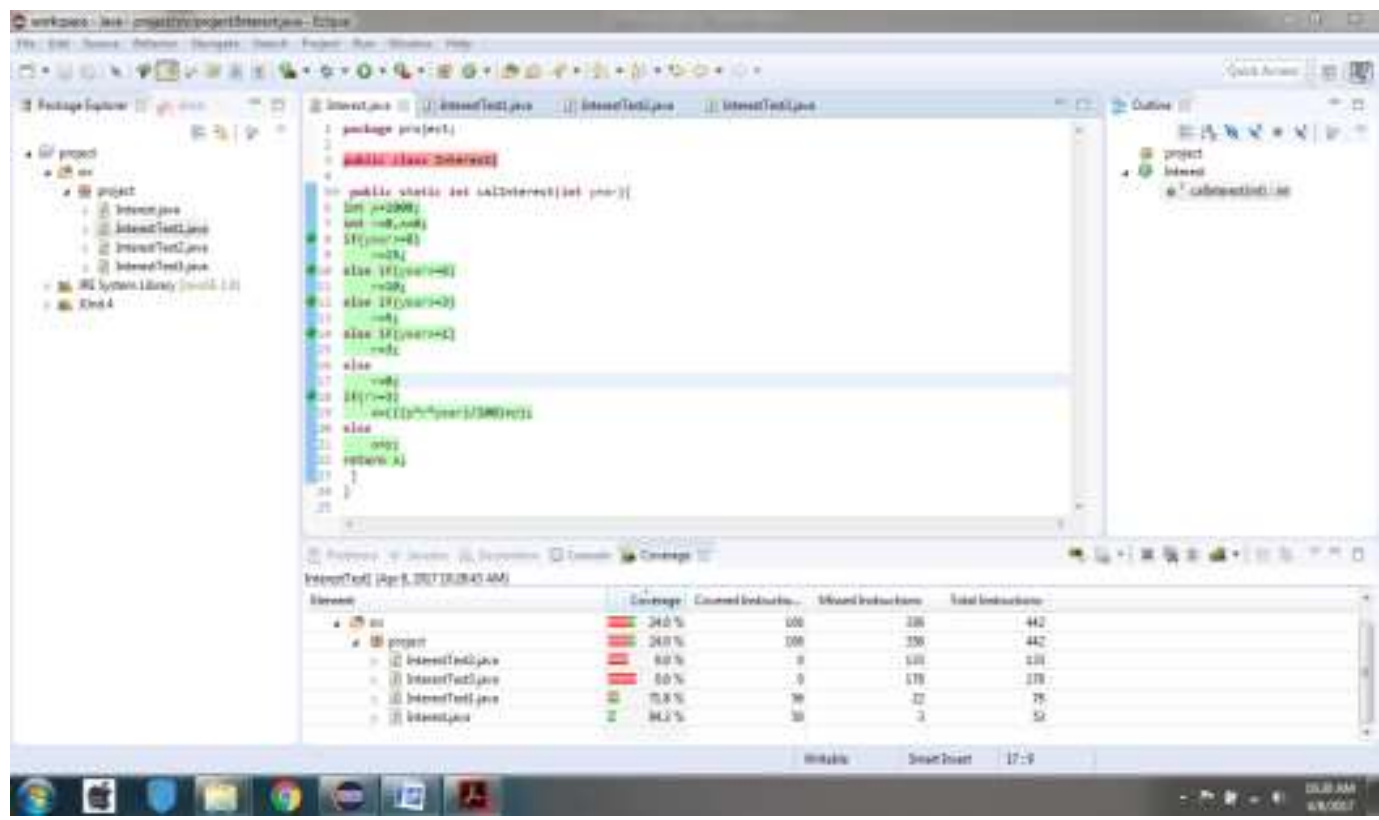

Figure 5.c.3. Coverage for the Test Sequence InterestTest1.java

\section{RESULTS AND DISSCUSSIONS}

The following result shows the coverage for the java program when different length test sequences are used. By this we can say that coverage will be more when optimized test sequences are used. 


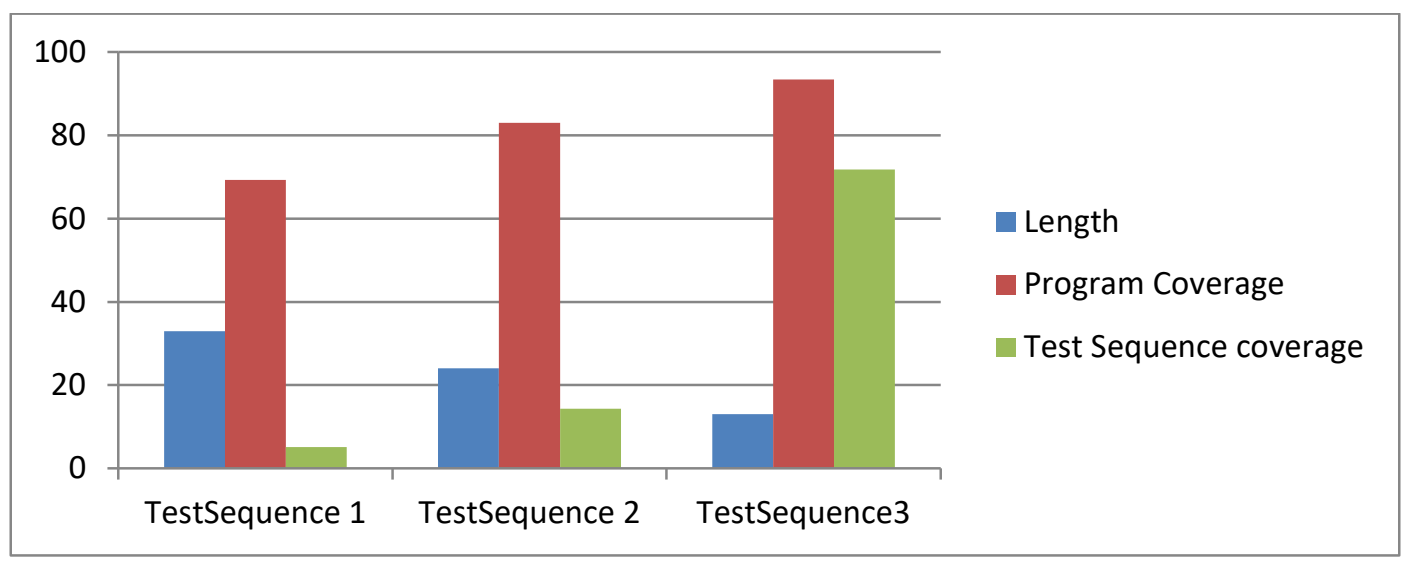

Fig. 6.1 Code Coverage in each case

\section{CONCLUSION FUTURE SCOPE}

In this we expressed that the length of test groupings keeping in mind the end goal to guarantee finish auxiliary scope in the product is the range less examined. we goes for breaking down the part of length of test successions for finish branch scope. we have taken a case java program and we made distinctive length test successions for the program utilizing Junit testing Frame. In the wake of composing we had run the test class and see breezed through and disappointment test cases. At that point by EclEmma device we will discover scope for each situation. At last we have demonstrated that scope will be high when upgraded successions are utilized. In this we have examined especially about branch scope. We have examined scope when diverse length test groupings are utilized. In future we can likewise amplify this for different sorts scope like practical, contingent, by utilizing some meta heuristic inquiry calculations like Hill climbing, Random Search, Evolutionary and Genetic calculations.

\section{REFERENCES}

[1] Andrea Arcuri "A Theoretical and Empirical Analysis of the Role of Test Sequence Length in Software Testing for Structural Coverage",IEEE VOL. 38, NO. 3, MAY/JUNE 2012.

[2] "Random Testing: Theoretical Results and Practical Implications" Andrea Arcuri, Member, IEEE IEEE TRANSACTIONS ON SOFTWARE ENGINEERING, VOL. 38, NO. 2, MARCH2012

[3] Arcuri, "Longer Is Better: On the Role of Test Sequence Length in Software Testing," Proc. IEEE Int'1 Conf. Software Testing, Verification and Validation, 2010

[4] "Are Longer Test Sequences Always Better? - A Reliability Theoretical Analysis" by Fevzi Belli1, Nevin Güler 2010 Fourth IEEE International Conference on Secure Software Integration.

[5] J.H. Andrews, A. Groce, M. Weston, and R.G. Xu, "Random Test Run Length and Effectiveness," Proc. IEEE/ACM Int'l Conf. Automated Software Eng., pp. 19-28, 2009.

[6] G. Fraser and A. Gargantini, "Experiments on the Test Case Length in Specification Based Test Case Generation," Proc. Int'l Workshop Automation in Software Test, 2009.

[7] B. Raveendranath Singh "Impact of Length of Test Sequence on Coverage in Software Testing", Special Issue of ICETEM Vol.2, No.6.

[8] G. Vivek, R.C. Narayanan "Minimization of Test Sequence Length for Structural Coverage Using VLR" IJRIT, Volume 1, Issue 5.

[9] "Fundamentals of software Engineering" Rajib Mal 3rd edition, Eastern Economy Edition, ISBN-97881-203-3819-7

[10] Software Engineering, A practitioner's Approach- Roger S. Pressman, 6th edition. McGraw-Hill International Edition.

[11] V. Kecman, "Learning and Soft computing", Pearson Education, India

[12] Principles of Soft Computing- S N Sivanandam, SN Deepa, Wiley India, 2011

[13] "The Unified Modelling Language Reference manual" Second edition by Ivar Jacobson, James R Rumbaugh, Grady Booch.

[14] "Unified Modeling User Guide" by Ivar Jacobson, James R Rumbaugh,Grady Booch.

[15] "Object Oriented Modeling and Design with UML" Second Edition by Michael R Blaha, James R Rumbaugh. 
[16] K. Koteswara Rao, G. S. V. P. Raju and S. Nagaraj, "Optimizing the software testing efficiency by using a genetic algorithm: a design methodology”, ACM SIGSOFT Software Engineering Notes, vol. 38, no.3, (2013), pp. 1-5.

[17] K. K. Rao and G. S. V. P. Raju, "Developing optimal directed random testing technique to reduce Interactive faults-systematic literature and design methodology", Indian Journal of Science and Technology, vol. 8, no. 8, (2015), pp. 715-719.

[18] K. Koteswara Rao and G. S. V. P. Raju, "Theoretical Investigations to Random Testing Variants and its Implications", International Journal of Software Engineering and Its Applications, vol. 9, no. 5, (2015),pp. 165-172.

[19] 19. J. Ratna Kumar, K. Koteswara Rao and D. Ganesh, "Empirical Investigations to Find Illegal and its Equivalent Test Cases using RANDOM-DELPHI", International Journal of Software Engineering and Its Applications, vol. 9, no. 11, (2015), pp. 107-116.

[20] 20 K. Koteswara Rao and G. S. V. P. Raju, "Random Testing: The Best Coverage Technique-An empirical Proof", International Journal of Software Engineering and Its Applications, vol. 9, no. 12, (2015), pp.115-122.

[21] 21. K. Koteswara Rao, "Measuring the Function Points from the Points of Relationships of UML", Computer and Electrical Engineering, ICCEE 2008. International Conference on IEEE, (2008). 